\title{
Charge-state dependence of kinetic electron emission induced by slow ions in metals
}

\author{
J. I. Juaristi, ${ }^{1}$ R. Díez Muiño, ${ }^{2}$ A. Dubus ${ }^{3}$ and M. Rösler ${ }^{4}$ \\ ${ }^{1}$ Departamento de Física de Materiales and Centro Mixto CSIC-UPV/EHU, Facultad de Ciencias Químicas, Universidad del País Vasco, \\ Apartado 1072, 20080, San Sebastián, Spain \\ ${ }^{2}$ Donostia International Physics Center DIPC, Paseo Manuel de Lardizabal 4, 20018, San Sebastián, Spain \\ ${ }^{3}$ Université Libre de Bruxelles, Service de Métrologie Nucléaire (Code Postal 165/84), 50 Avenue F. D. Roosevelt, \\ B-1050 Brussels, Belgium \\ ${ }^{4}$ Hahn-Meitner Institute Berlin, Glienicker Strasse 100, D-14109 Berlin, Germany \\ (Received 25 February 2003; published 15 July 2003)
}

\begin{abstract}
A calculation is performed in order to analyze the charge-state dependence of the kinetic electron emission induced by slow ions in metals. All stages of the emission process are included: the excitation of the electrons, the neutralization of the projectile during its passage through the solid, and the transport of the excited electrons from where they are created to the surface. It is shown that the number of excited electrons depends strongly on the ion charge state. Nevertheless, due to the fast neutralization of the ions within the escape depth of the excited electrons, no significant initial charge-state dependence is expected in the kinetic electron yield. This result is consistent with available experimental data.
\end{abstract}

DOI: 10.1103/PhysRevA.68.012902

PACS number(s): 34.50.Dy, 79.20.Rf

\section{INTRODUCTION}

In so-called ion induced electron emission, two different types of mechanism are customarily distinguished in regard to the origin of the excitation energy of the emitted electrons. In potential emission this energy originates in neutralization and deexcitation processes [1]. Since the number of originally unoccupied projectile bound states increases with the ion charge state $q$, the number of electrons emitted via this mechanism (the potential emission yield) is expected to increase with $q$. Indeed, this behavior has been clearly observed in experiments $[2,3]$.

The other electron ejection mechanism is kinetic electron emission, in which the excitation energy comes from the kinetic energy of the projectile. The electron excitation mechanism that gives rise to kinetic electron emission is responsible for the electronic stopping power of the target with respect to the incident ion. In recent work, the energy loss of slow multicharged ions $\left(v \leqslant v_{0}\right.$, the Bohr velocity) in metals has been measured [4-6]. These experiments showed a clear increase of the energy loss with $q$, which would lead to the prediction of a corresponding increase of the kinetic emission yield with $q$.

In order to obtain the kinetic emission yield from an experiment, one has to subtract the potential emission yield from the total measured yield. Considering the uncertainties inherent in this procedure (for instance, the dependence of the potential emission on the ion velocity is only approximately known), no significant charge-state dependence of the kinetic emission yield has been observed experimentally $[7,8]$. This shows that the previous prediction based only on the relation between the stopping power and kinetic emission is too simple and that further effects have to be taken into account.

One of the most important effects that must be taken into account is that the probability for electron excitation depends on time. The reason is that the electronic cloud that surrounds the ion evolves during its passage through the target.
The classical over-the-barrier model [9] predicts the survival of ion inner-shell vacancies until the instant at which the ion crosses the metal surface. When the ion enters the metal bulk, the inner-shell vacancies start to be filled until complete neutralization and relaxation of the ion is reached. Since the probability for electronic excitations depends on the stage of this process, the time scale on which it takes place is a crucial ingredient in the theoretical description of the electron yield.

The aim of this work is to present a consistent theoretical model which accounts for the relevant features that characterize the kinetic emission process. Attention is paid to (i) the electron excitation process, (ii) the neutralization of the ion projectile when traveling through the solid, and (iii) the transport of the excited electrons from the place where they are created to the vacuum. In order to make connection with the experimental data $[7,8]$, here we present results for $\mathrm{N}^{q+}$ projectiles traveling through an Au target. In a different part of this work the free electron gas model (FEG) is used to describe the valence band of Au. From the value of the plasmon energy of Au, we take $r_{s}=1.5$ (in atomic units) for the one-electron radius [10]. It will be shown that the fast neutralization of the ion prevents strong charge-state dependence of the kinetic yield.

The organization of the paper is as follows. In Sec. II the ingredients of the theoretical model are described, in Sec. III the results of the simulation are presented and discussed, and finally in Sec. IV the main conclusions of the work are summarized. Atomic units (a.u.) will be used unless it is otherwise stated.

\section{DESCRIPTION OF THE MODEL}

\section{A. Electron excitation}

The electronic excitations that govern the energy loss of an ion moving through a real metal have been successfully described by using the FEG model with an adequate electronic density $n_{0}$ [11]. Within this model a slowly moving 
ion loses energy, creating electron-hole pairs, i.e., some electrons are scattered from occupied electronic states below the Fermi energy to unoccupied states above the Fermi energy. If the energy of the excited electron surpasses the work function of the metal, the electron may eventually be emitted, giving rise to kinetic electron emission.

In this work we adopt a model developed in a previous paper [12] to calculate the energy and angle distribution of electrons excited via electron-hole pair creation in terms of the differential cross section for the scattering of electrons at the potential induced by the moving ion. The differential scattering cross section is calculated from a full phase shift calculation of the electron scattering. This approach is important because the slow ion represents a strong perturbation of the metal that cannot be treated within linear response theory [11]. Therefore, the ion-electron scattering must be calculated to all orders in the impurity charge. For the same reason it is necessary to perform a self-consistent calculation of the screened projectile induced potential. This is done using density functional theory (DFT) for a static impurity in a FEG [13].

Nevertheless, it is not trivial to define a charge state of an ion immersed in a FEG. No matter the character of the charged impurity, the metal electrons completely screen it. Therefore, the projectile together with its screening charge always represents a neutral object in the long range. The screening charge density cloud around an ion in a FEG consists of both scattering and bound components. In the previous work [12-15] in which this model was used to calculate the kinetic electron emission yield, only neutral or singly charged projectiles were considered. In these cases, the selfconsistent DFT potential for the ground state configuration (i.e., with all the low lying electronic bound states filled) was used. Here, in order to study higher projectile charge states, we consider configurations with vacancies in the projectile electronic bound states that we approximate by Kohn-Sham orbitals. For nitrogen we denote these different configurations by giving the occupation numbers of the $1 s, 2 s$, and $2 p$ orbitals as $(1 s, 2 s, 2 p)$. The natural way to establish a relationship between charge states in vacuum and electronic configurations inside the solid is to relate each charge state to the electronic configuration that has the same number of electrons in the inner shells. For instance, $\mathrm{N}^{5+}$ in vacuum gives rise to the $(2,0,0)$ configuration in the solid, $\mathrm{N}^{4+}$ to the $(2,1,0)$, etc. In an electron gas with $r_{s}=1.5$ the problem arises when one tries to put more than three electrons in the $L$ shell. In this case, the $2 p$ level is no longer bound in the solid and we denote this configuration $(2,2, *)$. Notice that this configuration is different from $(2,2,0)$, in which the $2 p$ level is bound but empty. As a matter of fact, the configuration $(2,2, *)$, which has all the bound levels filled, is the ground state of a $\mathrm{N}$ impurity in an electron gas. Therefore, we relate this configuration to the lowest charge state. This approach has been shown to be successful in explaining the measured increase of the energy loss with the charge state of slow $\mathrm{N}^{q+}$ scattered off an aluminum surface $[5,6]$.

In Fig. 1 we show the energy distribution (after performing angular integration) of the electrons excited by different configurations of $v=0.5$ a.u. $\mathrm{N}^{q+}$ ions traveling through an

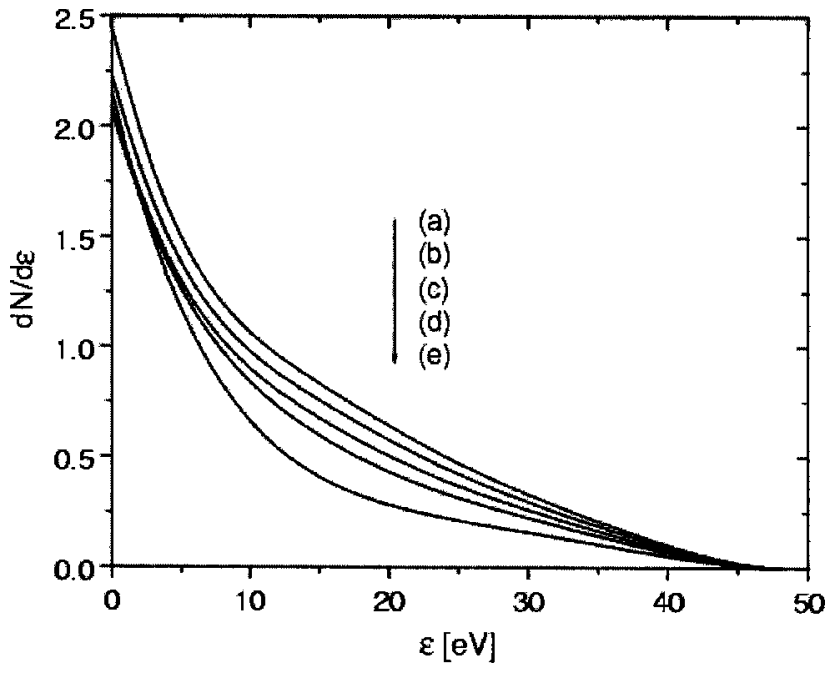

FIG. 1. Energy distribution of the electrons excited by different configurations of $v=0.5$ a.u. $\mathrm{N}$ ions traveling through an electron gas with $r_{s}=1.5$. Curves $a, b, c, d$, and $e$ correspond to the $(2,0,0)$, $(2,1,0),(2,2,0),(2,2,1)$, and $(2,2, *)$ configurations, respectively.

electron gas with $r_{s}=1.5$. A clear increase of the number of excited electrons is observed when depopulating the inner levels of the projectile, i.e., with increasing charge state of the ion. This behavior, consistent with the results obtained for the stopping power $[5,6]$, shows that the highly charged ions are more efficient in exciting conduction band electrons.

In Figs. 2(a) and 2(b) we present the angular distribution of electrons excited with energy $\varepsilon=20 \mathrm{eV}$ and $\varepsilon=6 \mathrm{eV}$ above the Fermi level. Results are shown for $v=0.5$ a.u $\mathrm{N}^{q+}$ ions with two different electronic configurations: the $(2,2, *)$ ground state configuration and the $(2,0,0)$ empty $L$-shell configuration. These distributions show a clear preference for electronic excitation in the direction of the moving ion. This behavior is even more pronounced for high excitation energies. The preponderance of the electronic excitation for the configuration corresponding to the highest charge state is also shown to be more important for high excitation energies. In principle, this would further enhance the charge-state effect due to the fact that the high energy electrons contribute in a larger amount than the low energy ones to the total yield because they produce a larger number of secondary lower energy electrons.

\section{B. Ion neutralization}

Several processes are involved in the neutralization of the ion under the surface: (a) quasiresonant capture from the target core levels $[16,17]$, which is important when the initial and final levels in the capture process are close in energy; (b) radiative capture, competitive for heavy ions [18]; and (c) Auger capture. In this work, we will focus our attention on the last process, which is the most effective one in the neutralization of $\mathrm{N}^{q+}$ ions in $\mathrm{Au}$. In an Auger capture process, an electron is captured from the valence band of the metal to a bound state in the ion. At the same time, an excitation is created in the medium. The excitation can be either an individual excitation (i.e., an electron-hole pair) or a collective 

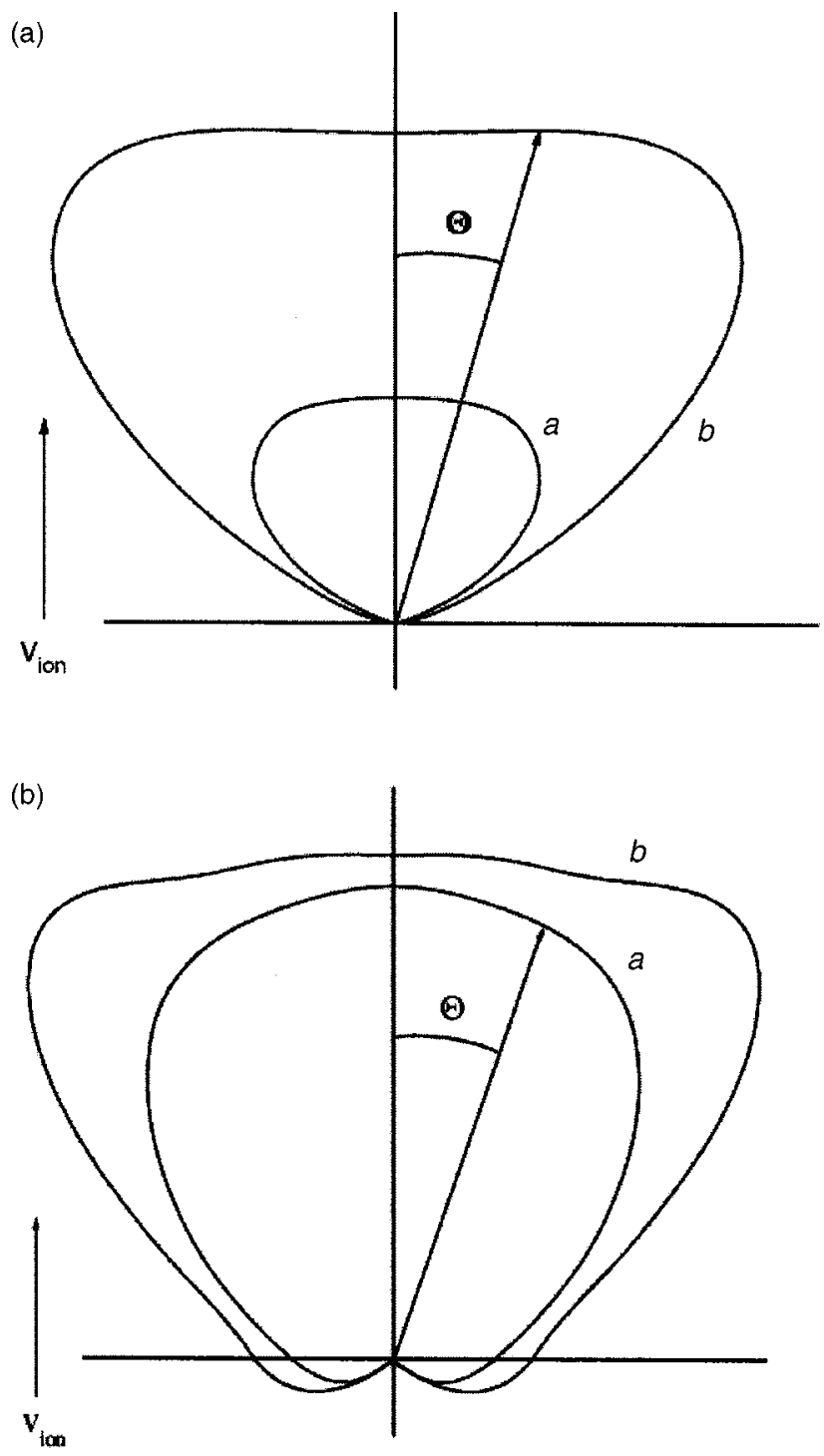

FIG. 2. Angular distribution of electrons excited with energy $\varepsilon$ $=20 \mathrm{eV}$ (a) and $\varepsilon=6 \mathrm{eV}$ (b) above the Fermi level, by $v$ $=0.5$ a.u. $\mathrm{N}$ ions in two different electronic configurations. Curves $a$ and $b$ correspond to the $(2,2, *)$ and $(2,0,0)$ configurations, respectively. $\Theta$ is the angle between the directions of the velocities of the excited electron and the ion.

excitation (i.e., a plasmon [19]). After the Auger capture process has taken place, the number of inner-shell vacancies of the ion is thus lowered.

The formalism used in the calculation of the Auger rates is described elsewhere $[18,20]$ and is only summarized here. The velocity of the ion is low enough to study the problem in the static situation. The valence band of the metal is described in the FEG approximation. Using first order of perturbation theory, the transition of the captured electron from the valence band of the metal to the ion bound state is calculated by a matrix element between two one-electron states. These one-electron states are approximated by Kohn-Sham orbitals, numerical solutions of the DFT calculation for the ion embedded in a FEG. The latter means that the strong perturbation introduced by the ion in the medium is included
TABLE I. $L$-shell Auger capture rates for a $\mathrm{N}$ ion inside a FEG of $r_{s}=1.5$. The rates are in atomic units. The first column of the table shows the initial and final configurations of the ion (see text for the details of the notation). The second column shows the Auger rate per spin state, and the third column is the total Auger rate (i.e., taking into account spin statistics).

\begin{tabular}{ccc}
\hline \hline \multicolumn{1}{c}{ Process } & Rate (per spin state) & Total Rate \\
\hline$(2,0,0) \rightarrow(2,1,0)$ & $8.19614 \times 10^{-3}$ & $1.639228 \times 10^{-2}$ \\
$(2,0,0) \rightarrow(2,0,1)$ & $1.63902 \times 10^{-2}$ & $9.834120 \times 10^{-2}$ \\
$(2,1,0) \rightarrow(2,2,0)$ & $9.36711 \times 10^{-3}$ & $9.367110 \times 10^{-3}$ \\
$(2,1,0) \rightarrow(2,1,1)$ & $1.42572 \times 10^{-2}$ & $8.554328 \times 10^{-2}$ \\
$(2,2,0) \rightarrow(2,2,1)$ & $1.04761 \times 10^{-2}$ & $6.285660 \times 10^{-2}$ \\
$(2,2,1) \rightarrow(2,2, *)$ & $8.67252 \times 10^{-3}$ & $4.336260 \times 10^{-2}$ \\
\hline \hline
\end{tabular}

in a self-consistent way in the calculation of the matrix elements.

Furthermore, the excitations in the medium are described by the imaginary part of the unperturbed medium response function. We use the wave-vector- and frequency-dependent random phase approximation (RPA) for the response function [21]. We remark here that the perturbation of the ion is not introduced in the calculation of the response function itself. Recent calculations [22] in which the ion perturbation is taken into account in the calculation of the medium excitations at the RPA level show that this effect modifies the Auger capture rates only slightly.

We show in Table I the Auger capture rates for a $\mathrm{N}$ ion inside a FEG of $r_{s}=1.5$. The $K$ shell of the $\mathrm{N}$ ion is full and the electron is captured to the $L$ shell of the ion. CosterKronig transitions from the $2 p$ level of the ion to the $2 s$ level of the ion usually are much faster than the Auger capture rates. Hence we consider that any electron captured to the $2 p$ level of the ion decays to the $2 s$ level before any other process can happen. The number of transitions to calculate is thus much reduced. The high absolute values of the calculated Auger capture rates (at least of the order of $10^{-2}$ a.u.) indicate that the neutralization of the $\mathrm{N}$ ion in the bulk of $\mathrm{Au}$ is an extremely fast process.

\section{Monte Carlo simulation}

In order to calculate the electron emission characteristics, we used a Monte Carlo simulation program. Both the incident projectile and excited electron trajectories are followed. Monte Carlo simulation for particle transport by classical trajectories has been thoroughly described in reference textbooks [23]. Details about the way in which ion induced electron emission can be simulated have been given in Ref. [24], for instance, for $\mathrm{MeV} \mathrm{He}$ ions incident on aluminum targets.

We briefly outline here the way in which electron emission for $\mathrm{N}^{q+}$ ions incident on Au targets has been described in the present work. First, we follow the incident projectiles, considering electron excitation as well as projectile neutralization (the incident ion is supposed to follow a straight-line trajectory without energy loss). Then we follow the excited electrons, including electron cascade and multiplication. 
For the incident projectile, two possible mechanisms can occur: either they excite electrons according to the model described in Sec. II A, or they undergo an Auger electron capture as described in Sec. II B. The two competing mechanisms for the incident $\mathrm{N}^{q+}$ ion give rise to a total "macroscopic" cross section (inverse total mean free path) $\Sigma_{t}$ $=1 / \lambda_{t}=\Sigma_{e}^{-}+\Sigma_{c} . \Sigma_{e}^{-}=1 / \lambda_{e}^{-}$is the "macroscopic" cross section (inverse mean free path) for electron excitation and $\Sigma_{c}=1 / \lambda_{c}$ is the "macroscopic" cross section for Auger electron capture. Starting at a given position (either the entrance position or the position where the ion has undergone its last collision) along the incident projectile trajectory with a given charge state $q$, the free path $L$ up to the next interaction for the incident projectile is sampled from the Poisson law $f(L)=1 / \lambda_{t} e^{-L / \lambda_{t}}$. Then the interaction (either an electron excitation or an Auger electron capture) is selected according to the respective probabilities of the two possible interactions $\left[P(\right.$ electron excitation $\left.)=\Sigma_{e}^{-} / \Sigma_{t}=\lambda_{c} /\left(\lambda_{e}^{-}+\lambda_{c}\right)\right]$. If the interaction is an electron excitation, the energy and angular direction of the excited electron are sampled from the energy differential and energy and angle differential electron excitation cross sections (for the given charge state of the projectile), respectively (see Figs. 1 and 2). The characteristics of the excited electrons are kept in the computer memory in order to follow these electrons when the ion trajectory is "finished" (see below). If the interaction is an Auger electron capture, $q$ is simply decreased by 1 . After considering the incident projectile interaction, its trajectory is further followed up to the next interaction. We stop following the incident ion when it is so deep in the target that excited electrons will no longer contribute to the electron emission.

The electrons excited along the ion trajectory are then followed. In this respect, electron interactions in metallic targets have been described thoroughly in Ref. [25]. In this case, two competing mechanisms have to be taken into account, i.e., inelastic collisions with the valence band described in the free electron gas model and elastic collisions with the ionic cores. The elastic collisions are usually described by considering a self-consistent electron-atom interaction potential. In the present work, we used phase shifts provided for Au targets by Heinz [26]. These phase shifts are calculated using a muffin-tin approximation with a suitable choice of the energy zero in the region between the muffintin spheres, taking into account the correct lattice structure of $\mathrm{Au}$. For the interaction of electrons with the outer-shell electrons, we will once again consider that the conduction electrons of $\mathrm{Au}$ can be described as a free electron gas with electron density parameter $r_{s}=1.5$. In this model, the energetic electrons excite additional electrons by binary collisions and plasmons. We will consider that plasmons decay by exciting one electron via an interband transition. A simple free electron density of states model has been used here (see [25], for instance). The electrons further excited by binary electron-electron collisions and by plasmon creation and decay will of course also contribute to the electron emission process. The elastic and inelastic mean free paths (as well as the total mean free path) for electrons in Au are shown in Fig. 3.

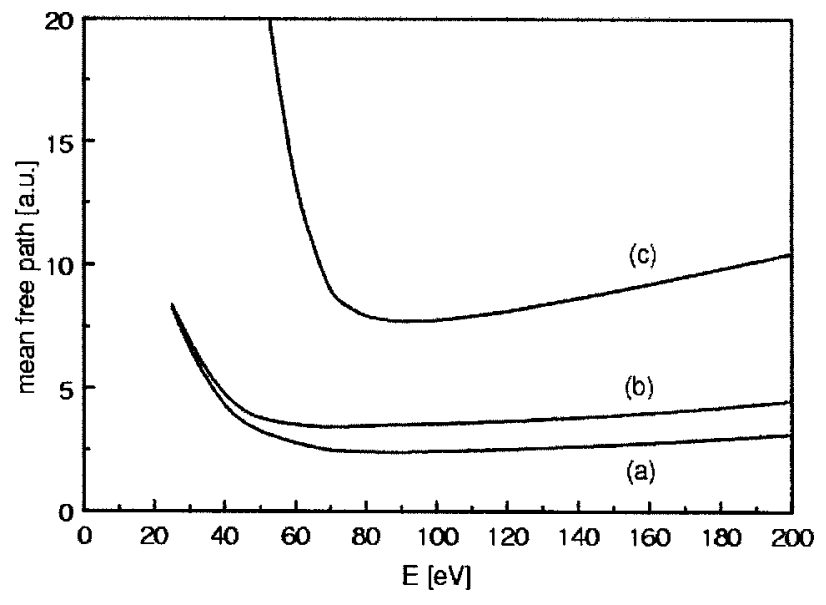

FIG. 3. Calculated total (curve $a$ ), elastic (curve $b$ ), and inelastic (curve $c$ ) mean free paths for electrons in $\mathrm{Au}$ as a function of their energy.

Therefore, the mean free paths for electron excitation in binary collisions $\lambda_{e e}=1 / \Sigma_{e e}$, plasmon excitation $\lambda_{\mathrm{pl}}$ $=1 / \Sigma_{\mathrm{pl}}$, and elastic collisions with ionic cores $\lambda_{\mathrm{el}}=1 / \Sigma_{\mathrm{el}}$ are calculated according to the models described above. Just as for the incident projectile, a total "macroscopic" cross section (inverse mean free path) $\Sigma_{\mathrm{el}, t}=1 / \lambda_{\mathrm{el}, t}=\Sigma_{e e}+\Sigma_{\mathrm{pl}}$ $+\Sigma_{\mathrm{el}}$ is used to sample the free path between the position where the electron has undergone its last collision (or where it was excited) and the next one. Once again, the interaction (a binary collision or a plasmon excitation or an elastic collision) is sampled according to the relative probability of each process. For all these mechanisms, a detailed description of the sampling of energy and angles for the incident electron (and for additional excited electrons) can be found in $[24,27]$. In the case of electrons, the trajectories are followed until they are emitted or until their energy is below the vacuum level. In this respect, the value of the work function that was used is $5.1 \mathrm{eV}$.

\section{RESULTS OF THE FULL SIMULATION}

In Fig. 4 we show the results of our simulation when the neutralization and relaxation of the $\mathrm{N}$ ion projectile when traveling through the solid is neglected. The number of electrons emitted per ion incident normal to the surface is plotted as a function of the ion velocity. A strong dependence of this quantity on the electronic configuration of the projectile is observed. For instance, at $v=0.5$ a.u. around seven electrons per ion are emitted when the ion travels in the most excited configuration considered here $(2,0,0)$, and only around 3.5 electrons when it travels in its ground state configuration. This behavior just reflects the strong dependence of the excitation function on ion configuration.

In Fig. 5 the results of the simulation including the neutralization of the $\mathrm{N}$ projectile via Auger processes are presented. The electron yield as a function of ion velocity is shown for different initial electronic configurations of the ion. It is observed that the dependence of the yield on the ion configuration is not so pronounced in this case. This is due to the fact that the ion relaxation takes place in a distance that is 


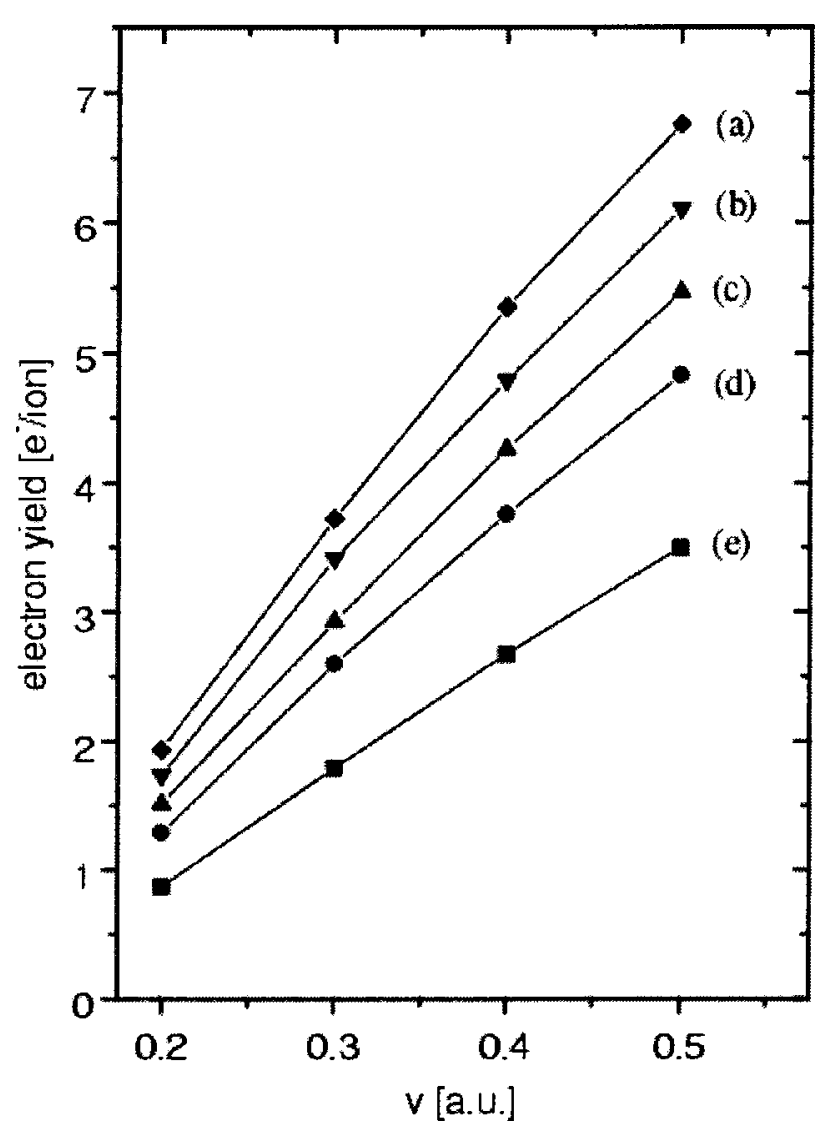

FIG. 4. Kinetic electron yield induced by Nions in different electronic configurations (charge states) traveling through $\mathrm{Au}$, as a function of the ion velocity. The charge state of the ion is frozen, i.e., the neutralization and relaxation of the ion along its path is neglected. Curves $a, b, c, d$, and $e$ correspond to the $(2,0,0)$, $(2,1,0),(2,2,0),(2,2,1)$ and $(2,2, *)$ configurations, respectively.

within the escape depth of the electrons. In order to observe this effect more clearly, we show in Table II for different initial electronic configurations the contribution to the total yield of the different excitation states in which the ion evolves during its neutralization-relaxation sequence. For instance, at $v=0.5 \mathrm{a}$.u. for an ion that is initially in the $(2,0,0)$ the total yield is 5.24. In this case, only 0.96 of the electrons emitted per ion (around $18 \%$ of the total) are excited by the ion in the $(2,0,0)$ configuration before it deexcites to the $(2,1,0)$ configuration. Additionally, 1.31 of the electrons emitted per ion (around $25 \%$ of the total) are excited by the ion in its ground state configuration at the end of its relaxation sequence.

If we invoke the above mentioned relationship between electronic configurations inside the solid and charge states in vacuum, our results show that no strong dependence of the kinetic electron yield on projectile charge state is expected for slow ions incident on a metal. This result is consistent with the measured data $[7,8]$. More precisely, in Fig. 3 of Ref. [8], is reported the experimentally obtained kinetic electron yield, after subtracting the potential contribution, for different charge states of $\mathrm{N}$ incident on $\mathrm{Au}$. For the higher charge states $\left(\mathrm{N}^{3+}, \mathrm{N}^{4+}\right.$, and $\left.\mathrm{N}^{5+}\right)$ a rather good qualitative and quantitative agreement can be observed between our re-

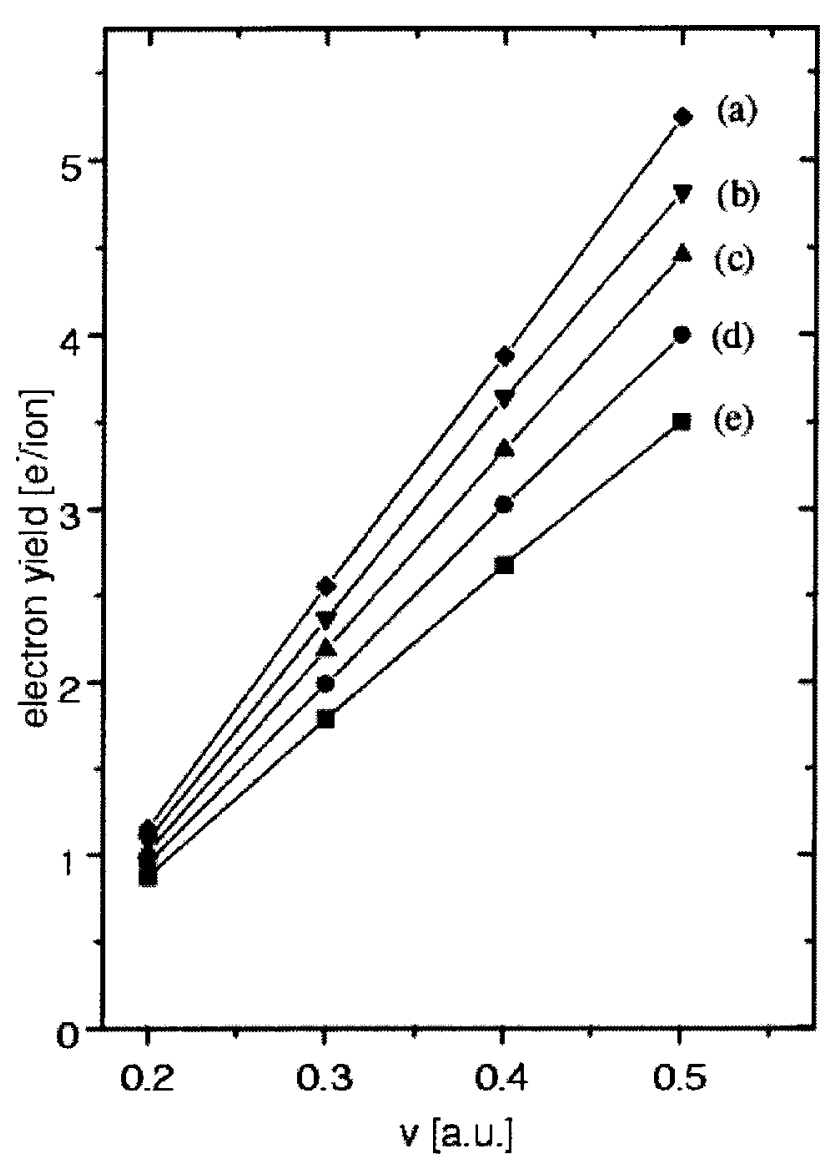

FIG. 5. Results of our complete simulation for the kinetic electron yield induced by $\mathrm{N}$ ions in different initial electronic configurations (charge states), as a function of the projectile velocity. The relaxation of the projectile from its initial excited state to the $(2,2, *)$ ground state via Auger processes is taken into account. Curves $a, b, c, d$, and $e$ correspond to the $(2,0,0),(2,1,0),(2,2,0)$, $(2,2,1)$, and $(2,2, *)$ initial electronic configurations, respectively.

sults and these data. Nevertheless, for the lower charge states $\left(\mathrm{N}^{+}\right.$and $\left.\mathrm{N}^{2+}\right)$ we find some discrepancies. In these cases, the measured yields are around 1-2 electrons per ion higher that the ones we obtain here, and also higher than the yields obtained for the higher charge states. This is probably due to a second contribution to the kinetic electron emission coming from the electron promotion to the continuum in projectile collisions with individual target atoms, which is expected to be important when the projectile carries a large number of bound electrons [7]. We remark that this effect further reduces the charge-state dependence of the electron yield because it follows an opposite dependence on the projectile charge as compared to the one we calculate here. Nevertheless, we stress that the direct electronic excitation of conduction band electrons is very strongly dependent on the ion configuration (charge state), as shown in Figs. 1 and 4. Our results show that the fast neutralization of the ion within the escape depth of the emitted electron is the reason why no strong dependence on the charge state is observed.

Finally, we want to emphasize that the results of our complete simulation (Fig. 5 and Table II) must be taken as higher limits for the charge-state dependence of the yield. When a 
TABLE II. Total yield and the contribution to it of the different electronic configurations of $\mathrm{N}$ ions as a function of the initial electronic configuration in which the ions enter the solid. The velocity of the ions is $v=0.5$ a.u. The target is Au.

\begin{tabular}{ccccccc}
\hline \hline Initial configuration & Total yield & $(2,0,0)$ & $(2,1,0)$ & $(2,2,0)$ & $(2,2,1)$ & $(2,2, *)$ \\
\hline$(2,0,0)$ & 5.24 & 0.96 & 0.80 & 1.05 & 1.12 & 1.31 \\
$(2,1,0)$ & 4.81 & & 1.18 & 0.99 & 1.29 & 1.35 \\
$(2,2,0)$ & 4.46 & & & 1.50 & 1.31 & 1.65 \\
$(2,2,1)$ & 4. & & & 2.12 & 1.88 \\
$(2,2, *)$ & 3.5 & & & & 3.5 \\
\hline \hline
\end{tabular}

highly charged ion approaches a metal surface it captures electrons into highly excited projectile states. These electrons are stripped when the ion enters the solid, contributing to the potential emission yield. Nevertheless, if some of these electrons are deexcited to the $L$ shell before entering the solid, i.e., before the electronic excitation process that gives rise to the kinetic electron emission is effective, one should consider the subsequent reduction of the initial charge state entering the simulation. This reduction of the initial excitation state would imply a reduction of the charge-state effect.

\section{CONCLUSIONS}

We have studied the charge-state dependence of the kinetic electron emission induced by slow $\mathrm{N}^{q+}$ ions incident on a Au target. We have shown that the electronic excitation depends strongly on the electronic configuration (charge state) of the ion. Nevertheless, since the neutralization and relaxation of the ion is a fast process, which takes place within the escape depth of the emitted electron, the total kinetic yield depends only slightly on the initial charge state. These results are consistent with available experimental data $[6,7]$ and expected to be valid for other projectile-target combinations.

\section{ACKNOWLEDGMENTS}

J.I.J. and R.D.M. acknowledge partial support from the Basque Departamento de Educación, Universidades e Investigación, the University of Basque Country UPV/EHU (Grant No. 9/UPV 00206.215-13639/2001), and the Spanish Ministerio de Ciencia y Tecnología (Grant Nos. BSM20010076 and MAT2001-0946). R.D.M. acknowledges partial support by the Gipuzkoako Foru Aldundia.
[1] P. Varga and H. P. Winter, Particle Induced Electron Emission II, Vol. 123 of Springer Tracts in Modern Physics (Springer, Berlin, 1991), p. 149.

[2] F. Aumayr and H. P. Winter, Commun. At. Mol. Phys. 29, 275 (1994).

[3] A. Arnau, F. Aumayr, P. M. Echenique, M. Grether, W. Heiland, J. Limburg, R. Morgenstern, P. Roncin, S. Schippers, R. Schuch, N. Stolterfoht, P. Varga, T. J. M. Zouros, and H. P. Winter, Surf. Sci. Rep. 27, 113 (1997).

[4] T. Schenkel, M. A. Briere, A. V. Barnes, A. V. Hamza, K. Bethge, H. Schmidt-Böcking, and D. H. Schneider, Phys. Rev. Lett. 79, 2030 (1997).

[5] J. I. Juaristi, A. Arnau, P. M. Echenique, C. Auth, and H. Winter, Phys. Rev. Lett. 82, 1048 (1999).

[6] J. I. Juaristi, A. Arnau, P. M. Echenique, C. Auth, and H. Winter, Nucl. Instrum. Methods Phys. Res. B 157, 87 (1999).

[7] H. Eder, F. Aumayr, and H. P. Winter, Nucl. Instrum. Methods Phys. Res. B 154, 185 (1999).

[8] H. Eder, M. Vana, F. Aumayr, H. P. Winter, J. I. Juaristi, and A. Arnau, Phys. Scr., T 73, 322 (1997).

[9] J. Burgdörfer, P. Lerner, and F. W. Meyer, Phys. Rev. A 44, 5674 (1991).

[10] D. Isaacson, New York University Document No. 02698 (1975) (National Auxiliary Publication Service, New York, 1975).

[11] P. M. Echenique, F. Flores, and R. H. Ritchie, in Solid State Physics: Research and Applications, Vol. 43, edited by $\mathrm{H}$.
Ehrenreich and D. Turnbull (Academic, New York, 1990), p. 229.

[12] J. I. Juaristi, M. Rösler, and F. J. García de Abajo, Phys. Rev. B 58, 15838 (1998).

[13] E. Zaremba, L. M. Sander, H. B. Shore, and J. H. Rose, J. Phys. F: Met. Phys. 7, 1763 (1977).

[14] J. I. Juaristi, M. Rösler, F. J. García de Abajo, H. Kerkow, and R. Stölle, Nucl. Instrum. Methods Phys. Res. B 135, 487 (1998).

[15] J. I. Juaristi and M. Rösler, Nucl. Instrum. Methods Phys. Res. B 157, 254 (1999).

[16] A. Arnau, R. Köhrbrück, M. Grether, A. Spieler, and N. Stolterfoht, Phys. Rev. A 51, R3399 (1995).

[17] N. Stolterfoht, A. Arnau, M. Grether, R. Köhrbrück, A. Spieler, R. Page, A. Saal, J. Thomaschewski, and J. Bleck-Neuhaus, Phys. Rev. A 52, 445 (1995).

[18] R. Díez Muiño, A. Salin, N. Stolterfoht, A. Arnau, and P. M. Echenique, Phys. Rev. A 57, 1126 (1998).

[19] D. Niemann, M. Grether, M. Rösler, and N. Stolterfoht, Phys. Rev. Lett. 80, 3328 (1998).

[20] R. Díez Muiño, N. Stolterfoht, A. Arnau, A. Salin, and P. M. Echenique, Phys. Rev. Lett. 76, 4636 (1996).

[21] J. Lindhard, K. Dan. Vidensk. Selsk. Mat. Fys. Medd. 28, 8 (1954).

[22] R. Díez Muiño, A. Arnau, A. Salin, and P. M. Echenique (unpublished). 
[23] M. H. Kalos and P. A. Whitlock, Monte Carlo Methods, Vol. I (Basics) (Wiley, New York, 1986); I. Lux and L. Koblinger, Monte Carlo Particle Transport Methods: Neutron and Photon Calculations (CRC Press, Boca Raton, 1991).

[24] A. Dubus, M. Rösler, and O. Benka, Math. Comput. Simul. 55, 37 (2001).

[25] M. Rösler, W. Brauer, J. Devooght, J. C. Dehaes, A. Dubus, M.
Cailler, and J. P. Ganachaud, Particle Induced Electron Emission I, Vol. 122 of Springer Tracts in Modern Physics (Springer, Berlin, 1991).

[26] K. Heinz (private communication).

[27] M. Cailler and J. P. Ganachaud, J. Microsc. Spectrosc. Electron. 5, 415 (1980). 